\title{
IoT Based Vehicle Emissions Monitoring and Inspection System
}

\author{
Prof. S.P. Bangal ${ }^{1}$, Gite Pravin E ${ }^{2}$, Ambhure Shankar G ${ }^{3}$, Gaikwad Vaibhav $\mathbf{M}^{4}$ \\ Assistant Professor, Electronics Department, PREC, Loni, India ${ }^{1}$ \\ Student, Electronics Department, PREC, Loni, India ${ }^{2,3,4}$
}

\begin{abstract}
The objective of this paper is to watch pollution on roads and track a vehicle that causes pollution, to unravel this downside, several countries and regions have already bestowed a series of emissions standards, meantime some ways has been developed, as well as update motor engine or up the standard of the gasoline. However, these actions have not caused hanging impact, as we tend to expect. During this system, Radio Frequency Identification (RFID) technology as an inexpensive and mature wireless communication methodology is adopted to gather and transmit emissions data of vehicles and Internet of Things (IoT) concept is proposed. Moreover, The RFID devices have to be put in on the traffic lights so reliable reading of emissions signals from a vehicle may be interrogated once the vehicles stop ahead of the red light .By applying the system; it is possible to smoothly realize green traffic network.
\end{abstract}

Keywords: Inspection system design, Internet of Things, Radio Frequency Identification, General Packet Radio System.

\section{INTRODUCTION}

The Internet of Things (IoT) may be a new idea that has attracted the attention of both academic and Industry. Internet of Things (IoT) is enforced as a network of interconnected objects, every of which might be addressed victimization unique id and communicates supported normal communication protocols. Carbon monoxide gas, gas oxides, are free once fuel is burned in an inside combustion engine and once emission of fuel are emitted through the vehicle pipe. Fuelvapours additionally escape into the atmosphere throughout supplying and once fuel vaporizes from engines and fuel systems caused by vehicle operation or weather condition. The consequences of those exhaust gases are delineated in additional detail below:

CO - Carbon Monoxides' reduces the blood's oxygen carrying capability, which might scale back the supply of oxygen to key organs. Extreme levels of exposure, like would possibly occur attributable to blocked flues in domestic boilers, is fatal. At lower concentrations, CO might create a health risk, significantly to those affected by heart disease.

NOx - Oxides of Nitrogen react within the atmosphere to make dioxide (NO2) which may have adverse effects on health, significantly among folks with disease. High levels of exposure are coupled with hyperbolic hospital admissions attributable to metabolic process issues, since long exposure might have an effect on respiratory organ work and increase the response to allergens in sensitive people.

\section{A. Radio-Frequency Identification}

Radio-frequency Identification (RFID) is that the wireless use of magnetic force fields to transfer information, for the needs of mechanically distinctive and trailing tags connected to things. The tags contain electronically hold on information. Some tags are high-powered by magnetic force induction from magnetic fields created close to the reader. Some sorts collect energy from the interrogating radio waves and act as a passive electrical device. Other types have a local power supply like electric battery and may operate at many meters from the reader.

\section{B. Internet of Things}

The Internet of Things (IoT) is the network of physical "objects" or "things" embedded with electronics, software, sensors and property to change it to attain bigger price and repair by exchanging information with the manufacturer, operator and alternative connected devices. Every factor is unambiguously diagnosable through its embedded automatic data processing system however is in a position to interoperate inside the present web infrastructure.

\section{General Packet Radio System}

General Packet Radio System is additionally called GPRS could be a third-generation step toward web access. GPRS is additionally called GSM-IP that is a Global-System Mobile Communications Internet Protocol because it keeps the 


\section{International Journal of Innovative Research in Electrical, Electronics, Instrumentation and Control Engineering}

\section{ISO 3297:2007 Certified}

Vol. 5, Issue 4, April 2017

users of these method on-line, permits to form voice calls, and access web on the go. Even Time-Division Multiple Access (TDMA) users enjoy this method because it provides packet radio access. GPRS additionally permits the network operators to execute associate degree Internet Protocol (IP) primarily based core design for integrated voice and knowledge applications, which will still be used and expanded for 3G services.GPRS supersedes the wired connections; this method hassimplified access to the packet knowledge networks like the web. The packet radio principle is used by GPRS to move user knowledge packets in an exceedingly structure method between GSM mobile stations and external packet knowledge networks. These packets are often directly routed to the packet switched networks from the GPRS mobile stations.

Within the current versions of GPRS, networks supported InternetProtocol (IP) just like the world internet and X.25 networks area unit supported.

D. Vehicle Emissions management

Vehicle emissions management is that the study of reducing the motorized vehicle emissions-emissions created by particularly combustion engines. Emissions of the many air pollutants are shown to own type of negative effects.

\section{E.Radio Frequency Identification (RFID) Tag}

An RFID tag may be a small radio device that is additionally remarked as an electrical device, smart tag, sensible label or radio barcode. The word electrical device comes from the words transmitter and communicator. The tag responds to a transmitted or communicated request for the information it carries. The tag contains an easy silicon semiconductor device (typically less than half a millimetre in size) connected to a small flat aerial and mounted on a substrate.

\section{F. Regarding Cloud}

The term Cloud refers to a Network or Internet. In alternative words, we will say that Cloud are some things that is present at remote location. Cloud will give services over network i.e. on public networks or on personal networks i.e. WAN, LAN or VPN.Applications like e-mail, net conferencing, customer relationship management (CRM), all run in cloud. Cloud term ordinarily utilized in Science and technologies as massive resource objects.

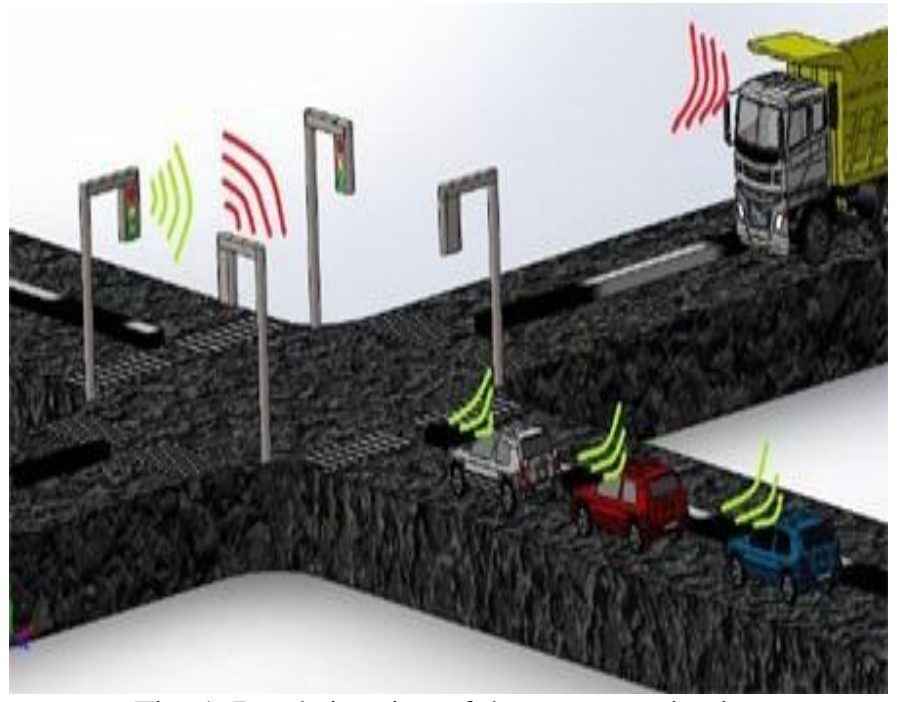

Fig. 1. Road situation of data communication

\section{EXISTING SYSTEM}

With the increasing number of automobiles, especially in some metropolis, such as Europe and America, it is very impending to resolve the problem of air pollution resulting from automobile exhaust gas.In Europe, air pollution has reached levels judged as hazardous to human health. To fight this problem, the motor emissions standards have been established and promoted in many developed countries for many years.

Furthermore, researchers have also developed some improved measures in vehicle engines or the quality of gasoline. However, these methods seem not to solve radically the emissions pollution problems. The motor emissions standard is very difficult to carry out in real- life. Although government forces all cars for testing or examining periodically as the local standard, the actual vehicle on-road emissions are usually much higher than those are, which are measured during the emission inspections. 


\section{III.PROPOSED SYSTEM}

The active RFID and the active RFID tag are designed to collect the emissions data from the vehicle exhaust system. RFID reader will receive emissions data when the vehicles, which are installed on RFID tag drive into, inspect range. However, most traffic lights in a city are just connected and controlled for shifting signals, which do not provide the capability of data transmission. Once the RFID reader receives the data from the tags, the most cost-effective way for data transmission is $3 \mathrm{G}$. It can steadily transmit these data to the control system.

In many cities, $3 \mathrm{G}$ data transmission is more popular compare to other wireless communication technologies and the price for $3 \mathrm{G}$ transmission is inexpensive. Collected data from ADC will send to ARM Microcontroller and then to RFID tag to Cloud Data Base Centre through GPRS and Cloud Web Server it may located in remote place, the GPRS Establish the connection with Cloud Web Server through Dongle.

\section{IV.DETAILED DESCRIPTION}

The implementation of the system has been described below:

A. Block Diagram

The blocks in the diagram depict the major components of the system and their interconnections.

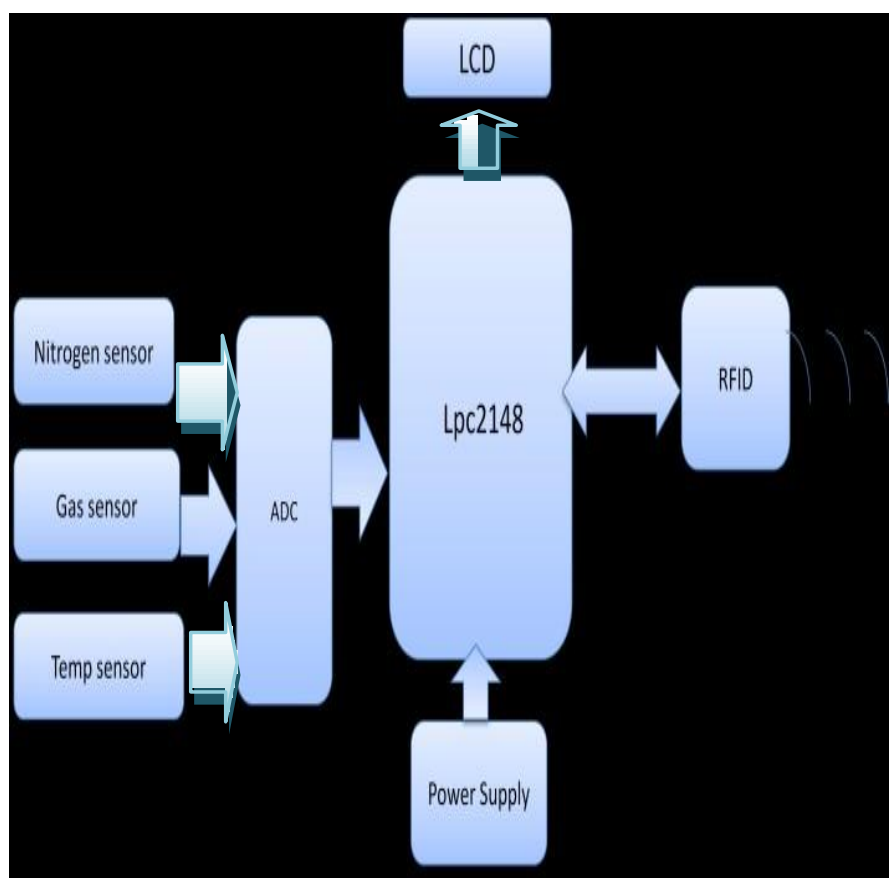

Fig. 2. Data Collection and Transmitter

1) Data Collection:

The active RFID the active RFID tag are designed to collect the emissions data from the vehicle exhaust system. Although active RFID tag has a lifetime, which is limited by the on-board power source, it is not an issue for WINS because there is already a power supply in every vehicle.

In a typical vehicle exhaust system, there are usually to built-in nitrogen \& gas, temperature sensors installed on the exhaust system. Therefore, the value is collected as the emissions data.In particular, the three sensors in the exhaust system of an automobile, the one is settled before the catalytic converter and the other one after it to test if the catalytic converter works well. To collect emissions data from the downstream sensor better, an analog-to-digital converter (ADC) is also designed to connect RFID tag. It is a device that uses sampling to convert a continuous quantity into a digital number and has been widely used for signal conversion problems. With ADC, the voltage signal (values) being outputted from the lambda sensors is first converted into digital signal, and then stored in the RFID tag directly via the wires. A prototype of the RFID tag with ADC is made and shown in Figure 2. 


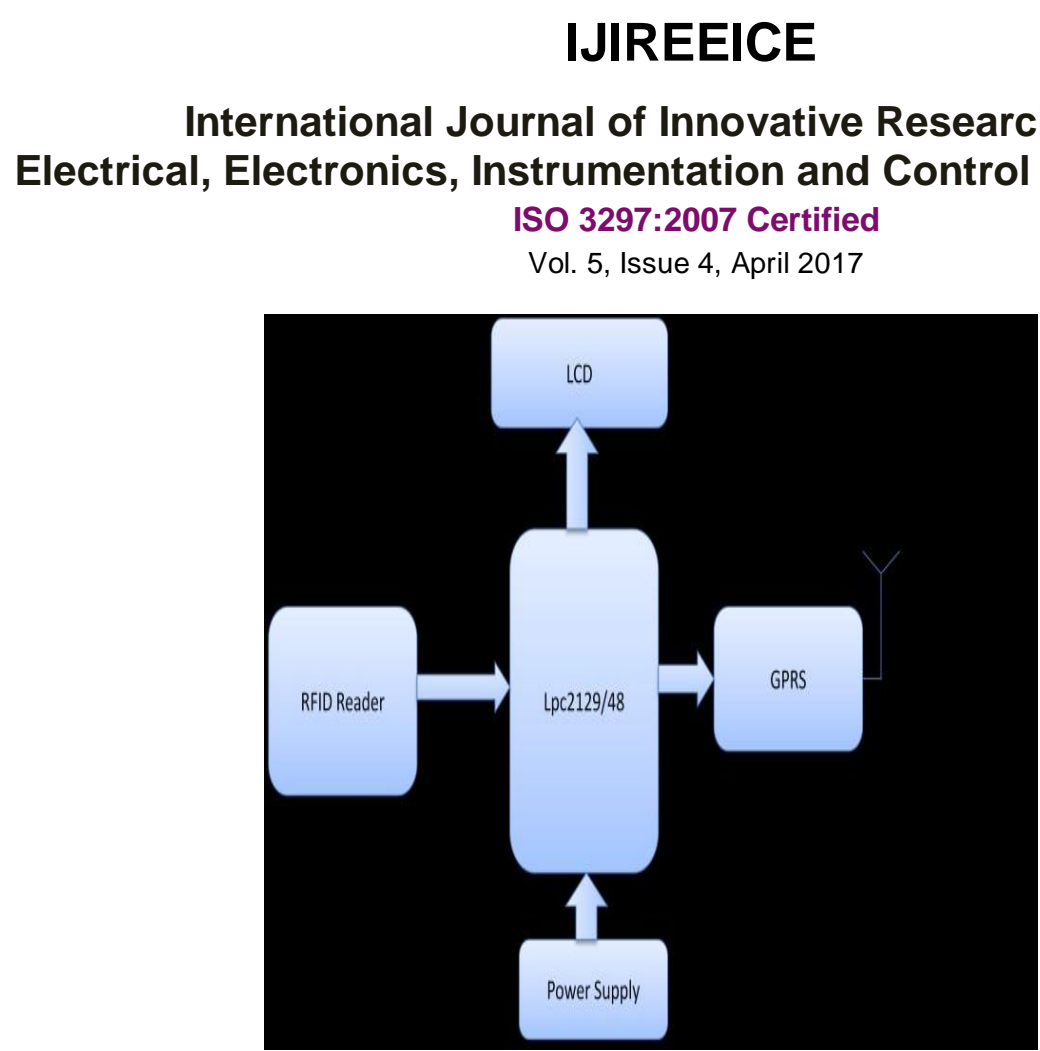

Fig. 3. RFID Reader and Transmitter to Cloud Server

2) Cloud Web Server and Cloud Data base Centre:

Collected data from ADC will send to Cloud Data Base Centre through GPRS and Cloud Web Server it may located in remote place, the GPRS Establish the connection with Cloud Web Server through Dongle, which is connected with Cloud Web Server. Then this cloud data can store into Data base centre can browse by any authenticated client from remote location, Cloud Web Server Act as Intermediate between Data Collection system, Cloud data base Centre and Cloud Web browser.

B. Flow Chart

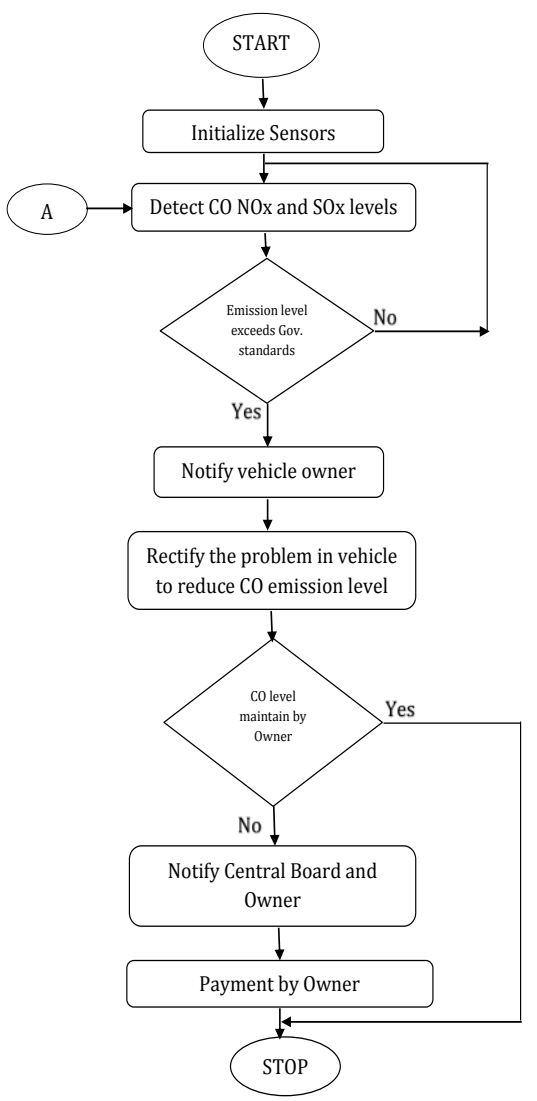

Fig. 4. Flow chart of Proposed System 
C. Implementation Methodology

1) Hardware Requirement:

- Two Microcontroller-32 bit LPC2148

- Power Supply

- Sensor 3 no's

- RFID Tag

- RFID Reader

- GPRS

- Dongle and Server PC

2) Software Requirement:

- Kiel micro vision

- Flash Magic

- Embedded C Program

- Drop box

D. Advantages

- Green environment can create in the city.

- Remotely data cloud access.

- Remotely data cloud store.

- To reduce the human inspection

\section{HARDWARE IMPLEMENTATION}

A. Transmitter Side

In this section, we would like to present effective use of Internet of Things to address the issue of vehicular pollution. Continuous monitoring of air quality is necessary to find out level of pollution and presence of certain harmful pollutants. Three sensors are placed to detect temperature, gas, nitrogen. Whenever the sensor sense abrupt rise in pollution, search is initiated for concerned RFID tags, i.e. vehicles causing pollution are identified using the RFID tag attached on them. Here we are using ZIGBEE to transmit data and RFID reader and the RFID tag are designed to collect data. This process will display in LCD.

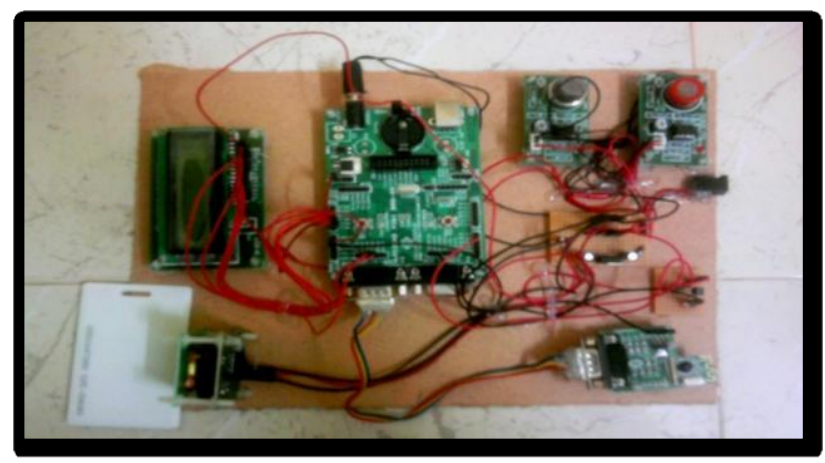

Fig. 5. Transmitter Side of Proposed System

B. Receiver Side

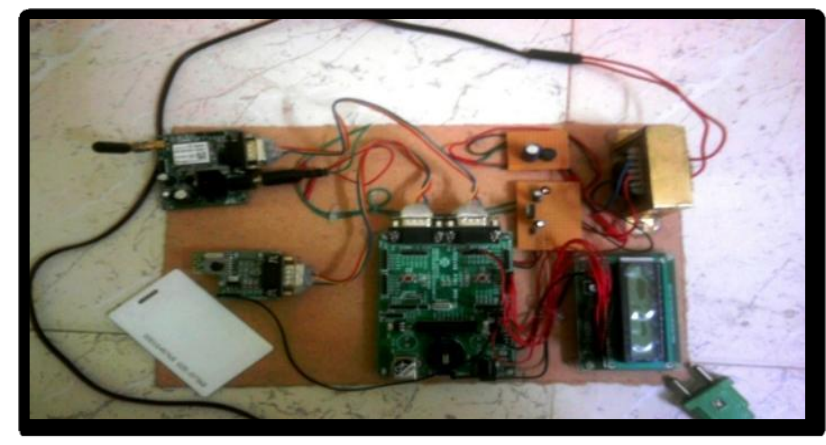

Fig. 6. Receiver Side of Proposed System 


\section{IJIREEICE

The RFID readers identify corresponding tag and transmit the same via the GPRS modem to the server. This framework also generates alert when pollution level increases. Authorities may take appropriate actions accordingly. Collected data from ADC will send to Cloud Data Base Centre through GPRS and Cloud Web Server it may located in remote place, the GPRS Establish the connection with Cloud Web Server through Dongle, which is connected with Cloud Web Server.

C. Experimental Results

1) RFID Based Emission Detection Using Cloud Computing:

Here we detect the Gas \& Nitrogen value from that we know the smoke is emitting or not. Hence, the gas \& Nitrogen sensors are digital sensors, when we detect ' 1 ' as output it means it is polluted (Gas, Nitrogen) and the analog sensor, which is Temperature, will detect the engine temperature.

2) Database:

For the particular car tag, we remotely data could be stored like time, temp, gas, Nitrogen, decision. Decision means address of the particular vehicle.

\section{CONCLUSIONS}

RFID technology, as one of the enabling technologies of IoT (Internet of Things).This technology employed to develop the information system. RFID reading can be interrogated along with the corresponding tag ID through a wireless connection among traffic lights and vehicles. This will maintain the database and graph for the data by monitoring the emissions data, the engine health can be easily inspected and examined.

\section{REFERENCES}

[1] Bengt Johansson, "Will new technology be sufficient to solve the problem of air pollution caused by Swedish road transport?" Transport Policy, Vol. 5, pp. 213-221, October 2012.

[2] D. S. Eisinger, "Evaluating inspection and maintenance programs: A policy-making framework", Journal of the Air \& Waste Management Association, vol. 55, pp. 147-162, Feb 2009.

[3] D.P. Chock, S.L. Winkler, T.Y. Chang, S.J. Rudy, and Z.K. Shen,"Urban ozone air quality impact of emissions from vehicles using reformulated gasolines and M85", Atmospheric Environment, Vol. 28, pp. 2777-2787, September 2012.

[4] F. Moreno, M. Muñoz, J. Arroyo, O. Magén, and C. Monné, I. Suelves, "Efficiency and emissions in a vehicle spark ignition engine fuelled with hydrogen and methane blends", International Journal of Hydrogen Energy, Vol. 37, Issue 15, pp. 11495-11503, August 2010.

[5] L. Yan, Y. Zhang, L. T. Yang, and H. Ning, "The Internet of things: from RFID to the next- generation pervasive networked systems", Auerbach Publications, 2015.

[6] T. N. Hubbard, "Using inspection and maintenance programs to regulate vehicle emissions", Contemporary Economic Policy, vol. 15, pp. 5262,2014 . 